Ophthalmologe $2021 \cdot 118: 885-892$ https://doi.org/10.1007/s00347-021-01470-w Angenommen: 8. Juli 2021

Online publiziert: 18 . August 2021

(c) Springer Medizin Verlag $\mathrm{GmbH}$, ein Teil von Springer Nature 2021

Die COVID-19-Pandemie hat im Jahr 2020 und auch in großen Teilen von 2021 alle Teile des öffentlichen und privaten Lebens weltweit mitbestimmt. Vielleicht mehr als jeder andere Lebensbereich war und ist die Medizin davon betroffen: angefangen von der direkten Versorgung der Betroffenen über die veränderten klinischen Abläufe in Krankenhäusern hin zu Einschränkungen bei der Behandlung von akut und chronisch Erkrankten, die nicht direkt vom Virus betroffen waren. Über mehr als 1 Jahr wurden aufgrund von verschärften hygienischen Maßnahmen Abstandsregeln und auch aus Angst vor Ansteckung unter medizinischem Personal und Patienten gleichermaßen weniger Patienten behandelt, mussten elektive Eingriffe aufgeschoben oder ganz abgesagt werden. Durch diese Beschränkungen hat die Telemedizin auch in der Ophthalmologie an Interesse gewonnen.

Telemedizinische Ansätze waren vor der SARS-CoV-2-Pandemie oft vornehmlich auf die bessere medizinische Versorgung von unterprivilegierten, unterversorgten Bevölkerungsgruppen in Entwicklungs- und Schwellenländern ausgerichtet. Ziel dieser Ansätze ist oftmals, kostengünstige und v. a. robuste,

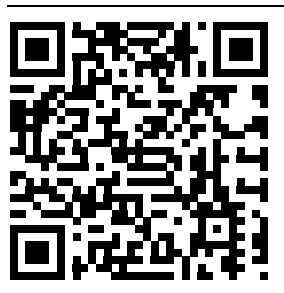

QR-Code scannen \& Beitrag online lesen

Lars Choritz ${ }^{1,2} \cdot$ Michael Hoffmann $^{1} \cdot$ Hagen Thieme ${ }^{1}$

${ }^{1}$ Universitätsaugenklinik, Magdeburg, Deutschland

${ }^{2}$ Medizinische Fakultät, Otto-von-Guericke Universität, Universitätsaugenklinik, Magdeburg, Deutschland

\title{
Telemedizinische Ansätze in der Augenheilkunde in Zeiten von COVID-19
}

mobile Alternativen für die hoch spezialisierten medizinischen Geräte zu schaffen, die in der westlichen Welt fast jederzeit zugänglich sind. Im Bereich der Ophthalmologie wurde z.B. das "portable eye examination kit" (peek, Peek Vision Ltd., Hertfordshire, England) entwickelt, das unter anderem Smartphone-basierte Fundusfotografie ermöglicht (www.peekvision.org) [15].

Durch die Pandemie ist in den vergangenen anderthalb Jahren jedoch auch in der westlichen Welt eine relative Unterversorgung entstanden, nicht etwa durch fehlende Verfügbarkeit, sondern v. a. durch den eingeschränkten $\mathrm{Zu}$ gang $\mathrm{zu}$ medizinischem Equipment, medizinischen Maßnahmen oder medizinischem Personal. Dies hat zu einem verstärkten Interesse an der Entwicklung und Nutzung von telemedizinischen Anwendungen weltweit geführt, auch in der Ophthalmologie. Die - Abb. 1 zeigt den sprunghaften Anstieg der wissenschaftlichen Veröffentlichungen $\mathrm{zu}$ den Suchworten "telemedicine“ und „ophthalmology“ im Jahr 2020. Allein 108 Publikationen findet man zusätzlich für das Jahr 2020 und 55 für das Jahr 2021 (Stand April 2021), wenn das Suchwort „Covid“ ergänzt wird.

Dieser Beitrag zielt auf eine orientierende Übersicht der telemedizinischen Ansätze, die es in der Ophthalmologie derzeit gibt, welche Vor- und Nachteile sie in Zeiten eingeschränkten Zugangs zu regulärer Versorgung bieten und wie sie weiterentwickelt werden könnten, um auch über die COVID-19-Pandemie hinaus von Nutzen für Patienten und Oph- thalmologen zu sein. Dabei wird auf 4 wesentliche Aspekte eingegangen:

1. Screeningmethoden zur (Früh-)Erkennung von Erkrankungen außerhalb der Praxis/Klinik,

2. Monitoring bei chronischen Augenerkrankungen,

3. therapeutische und rehabilitative Ansätze,

4. ophthalmologische Videosprechstunden.

\section{Screeningmethoden zur}

(Früh-)Erkennung von

Erkrankungen außerhalb der

Praxis/Klinik

"Smart devices“ (Smartphones und Tablet-Computer) bieten aufgrund ihrer hohen Bildschirmauflösung die Möglichkeit, einfache ophthalmologische Funktionstests durchzuführen. In den App Stores der großen SmartphoneAnbieter findet sich dementsprechend eine kaum überschaubare Anzahl „medizinischer" Apps aus dem Bereich der Ophthalmologie, die sich zumindest theoretisch als telemedizinische Screeningwerkzeuge einsetzen ließen. Diese reichen von Nahvisustests über Farbsehtests hin $\mathrm{zu}$ professionell anmutenden Optometrieanwendungen, die mehrere Funktionen in sich vereinen.

Smartphone-Anwendungen dieser Art fallen potenziell unter das Medizinproduktegesetz (MPG), insbesondere wenn sie „dazu bestimmt [sind], Informationen für die Erkennung, Diagnose, Überwachung oder Behandlung von physiologischen Zuständen, Gesundheitszuständen, Krankheitszuständen 


\section{Leitthema}

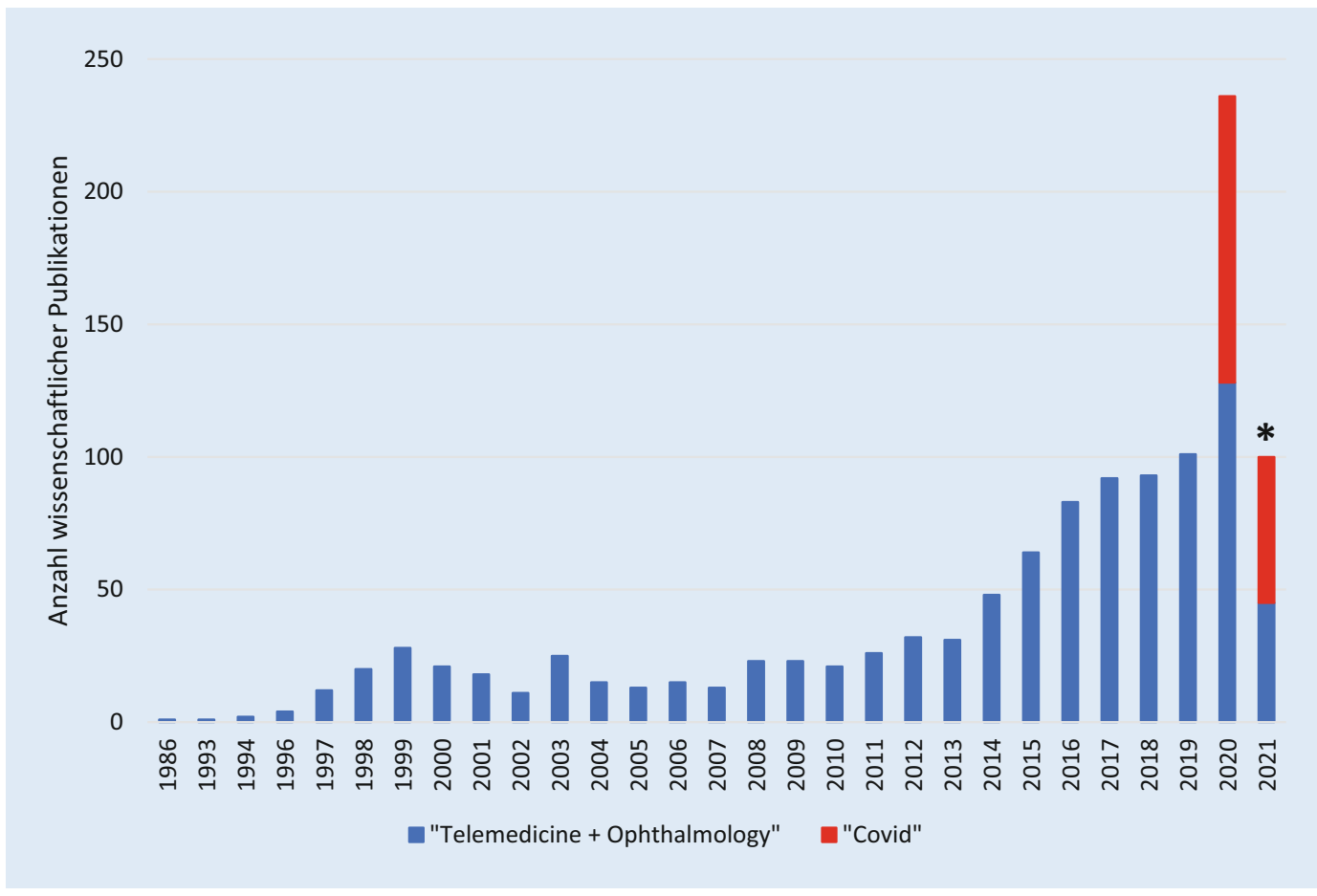

Abb. $1 \triangleleft$ Anzahl der jährlichen wissenschaftlichen Publikationen zu den Suchworten "Telemedicine", „Ophthalmology" und "Covid" bei PubMed. Stern Stand April 2021

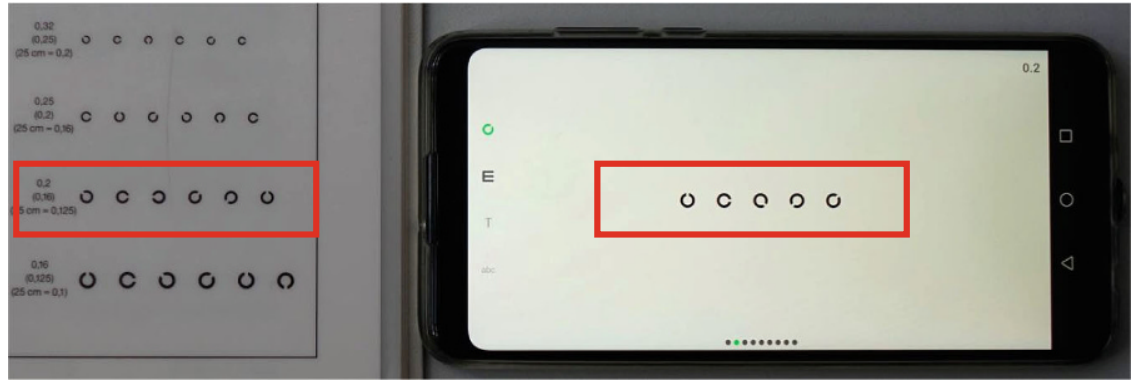

Abb. 2 \ Beispiel einer App zur Prüfung des Nahvisus. Die Optotypen auf dem Smartphone sind ca. $15 \%$ größer als in der Printversion. Die Vergleichbarkeit wird darüber hinaus durch die Bildschirmhelligkeit und den Kontrast beeinflusst

oder angeborenen Missbildungen $\mathrm{zu}$ liefern" (Auszug aus den Klassifizierungsregeln des Anhangs IX der Richtlinie 93/42/EWG). Eine kursorische Suche fand jedoch nur bei einem Bruchteil der Anwendungen Hinweise auf eine CEZertifizierung oder Klassifizierung nach dem MPG. Darüber hinaus ließen sich im App-Store und oft auch auf den Internetseiten der Anwendungsentwickler in der Regel keine Angaben darüber finden, ob und wie diese Tests validiert wurden. Gelegentlich fand sich der Hinweis, dass es sich bei der App nicht um ein Medizinprodukt handele. Dieser entbindet jedoch nicht notwendigerweise von der Einstufung im Sinne des MPG.

Es verwundert deshalb nicht, dass die Qualität der frei verfügbaren Apps ex- trem unterschiedlich ausfällt. So zeigten Steren et al. kürzlich, dass die Größe der Optotypen in Visustest-Apps oft von der Bildschirmgröße des Geräts abhängt und teilweise bis zu $400 \%$ von der korrekten Größe abweichen kann [20]. Die • Abb. 2 zeigt ein Beispiel mit relativ guter Übereinstimmung einer App mit Standardoptotypen. Für beide ist ein Leseabstand von $40 \mathrm{~cm}$ angegeben, die Landolt-Ringe auf dem Mobiltelefon sind nur geringfügig größer als auf der vergleichbaren Printversion. Die zuverlässigsten Ergebnisse zeigen hier solche Anwendungen, die einen Größenindikator zur „Kalibration" verwenden. Die große Mehrzahl der Smartphone-Visustests in den AppStores ist nicht erkennbar validiert oder zertifiziert.
Auch Smartphone-Farbsehtests sind derzeit nur mit Vorsicht zu verwenden. Die 0 Abb. 3 zeigt die Abhängigkeit der Farbdarstellung von den Bildschirmeinstellungen. Idealerweise sollten für computerbasierte Farbsehtests farbkalibrierte Monitore verwendet werden. Da diese in der Regel sehr teuer sind, sollte ein computerbasierter Farbsehtest zumindest über ein Testbild verfügen, anhand dessen Bildschirmhelligkeit, Kontrast und Farbsättigung grob eingestellt werden können. Andernfalls sind verlässliche Tests nur schwer durchführbar.

Eine Sonderstellung unter den frei verfügbaren Screening-Apps nehmen sog. Foto-Screening-Apps ein [1]. Der "CRADLE White Eye Detector" beispielsweise durchsucht systematisch sämtliche Fotos auf dem Mobiltelefon/ Tablet nach auffälligen Fundusreflexen und Leukokorie. Die Bilderkennung basiert auf "deep learning" mittels neuronaler Netzwerke anhand von Trainingsbildern. Munson et al. zeigten in einer retrospektiven Analyse von mehr als 50.000 Familienfotos aus dem Leben von 20 Kindern mit Leukokorie (darunter 15 Patienten mit Retinoblastom und 5 Patienten mit Morbus Coats oder Katarakt) sowie 20 augengesunden Kindern, dass die App in $80 \%$ der Fälle 
eine Leukokorie durchschnittlich bereits mehr als 1 Jahr vor der tatsächlichen Diagnosestellung erkannt hätte (95\%Konfidenzintervall 0,4 bis 2,3 Jahre) bei allerdings geringer Spezifität (20\%) [18]. Diese und vergleichbare Foto-ScreeningApps könnten somit potenziell lebensrettende Hilfsmittel sein. Die Nutzung von künstlicher Intelligenz (K.I.) zur Erkennung von Pathologien hat bereits in der Verarbeitung von Fundusbildern und OCT(optischer Kohärenztomographie)Volumenscans in die Ophthalmologie Einzug gehalten. Denkbar wäre auch die Verwendung von K.I. zur Erkennung anderer ophthalmologischer Erkrankungen, wie z. B. endokriner Orbitopathie, oder seltener syndromaler Erkrankungen mit Augenbeteiligung anhand von Fotos. Tatsächlich wird auf dem Gebiet der seltenen und syndromalen Erkrankungen aktiv geforscht, und Deep-learning-Algorithmen können zum Teil bereits jetzt bestimmte seltene Erkrankungen mit hoher Wahrscheinlichkeit identifizieren [10, 17].

\section{》) Deep-learning-Algorithmen können seltene Erkrankungen mit hoher Wahrscheinlichkeit identifizieren}

Zugleich bergen solche auf Bilderkennung mittels K.I. basierende Screeningtools jedoch auch erhebliches Missbrauchspotenzial. Auf große Bilddatenbanken sozialer Medien angewandt, könnte in großem Umfang Verletzung von Persönlichkeitsrechten und Datenschutz erfolgen. Es ist daher zu diskutieren, ob solche Anwendungen nicht grundsätzlich in der Hand von Fachärzten oder spezialisierten Zentren bleiben sollten.

Auch für die „einfacheren“, oben genannten Screening-Apps ist eine augenärztlich kontrollierte Anwendung zu überlegen, um so z.B. Fehlinterpretationen von erhobenen Messwerten vorzubeugen. So ist beispielsweise eine validierte optometristische SmartphoneAnwendung zur Testung von Nahvisus, Kontrastsehen und Metamorphopsien in Frankreich nur auf Rezept verfügbar,

Ophthalmologe 2021 · 118:885-892 https://doi.org/10.1007/s00347-021-01470-w

(c) Springer Medizin Verlag GmbH, ein Teil von Springer Nature 2021

\section{Choritz $\cdot$ M. Hoffmann $\cdot H$. Thieme}

\section{Telemedizinische Ansätze in der Augenheilkunde in Zeiten von COVID-19}

\section{Zusammenfassung}

Hintergrund. Während der COVID-19Pandemie war die augenärztliche Versorgung teilweise eingeschränkt.

Fragestellung. Es wird ein Überblick über bereits verfügbare oder noch in Entwicklung befindliche teleophthalmologische Anwendungen zur besseren Versorgung während der Pandemie gegeben.

Material und Methode. Es erfolgten die Auswertung aktueller wissenschaftlicher Publikationen, die Analyse von frei erhältlichen Screeninganwendungen in App-Stores mobiler Endgeräte sowie telemetrischer Medizinprodukte für Monitoringanwendungen und die Diskussion der Ansprüche an eine ophthalmologische Videosprechstunde. Ergebnisse. Das gesteigerte Interesse an teleophthalmologischen Anwendungen spiegelt sich in einem deutlichen Anstieg von Publikationen zum Thema wider. Frei erhältliche Anwendungen für Smartphones und Tablets sind in der Regel nicht validiert und weisen teils starke Abweichungen von etablierten Standardtests auf. Telemedizinische Medizinprodukte zum Home-Monitoring bei chronischen Anwendungen bergen großes Potenzial, müssen sich aber erst im klinischen Alltag durchsetzen.

Schlussfolgerungen. Es besteht ein hoher Bedarf an systematischer Analyse, Weiterentwicklung und Validierung von telemedizinischen Anwendungen und Medizinprodukten für die Ophthalmologie, um das Potenzial der Telemedizin gewinnbringend zu nutzen und in einer ophthalmologischen Videosprechstunde zu bündeln.

Schlüsselwörter

Selbstmessung · Videosprechstunde . Künstliche Intelligenz · COVID-19-Pandemie · Home-Monitoring

\section{Telemedical applications in ophthalmology in times of COVID-19}

\section{Abstract}

Background. During the coronavirus disease 2019 (COVID-19) pandemic access to and utilization of ophthalmologic healthcare providers was partially restricted.

Objective. This article provides an overview of already available tele-ophthalmologic applications for better care during the pandemic as well as those still under development.

Material and methods. The study included an analysis of current scientific publications, analysis of unrestricted screening applications in smart device app stores as well as telemetric medical products specifically designed for home monitoring and discussion of the requirements of an integrated ophthalmologic video consultation.

Results. There is significant interest in teleophthalmologic applications and devices as evidenced by a rise in the number of relevant publications. Freely available screening tests for smart phones and tablets are as a rule currently not validated and show significant discrepancies from established standard tests. Telemetric medical devices show great potential for home monitoring in chronic ophthalmologic diseases but must first become established in the clinical routine. Conclusion. There is an unmet need for systematic analysis, development and validation of telemedical applications and medical products for ophthalmology in order to advantageously utilize the potential of telemedicine and to incorporate this into an ophthalmologic video consultation.

\section{Keywords}

Self-measurement - Video consultation . Artificial intelligence COVID-19 pandemic . Home monitoring nicht jedoch frei im App-Store erhältlich [3].

Auch in Deutschland gibt es bereits erstattungsfähige Apps, die die Ansprüche an eine sog. ,digitale Gesundheitsanwendung“ (DiGA) erfüllen. Es handelt sich dabei um CE-zertifizierte Anwendungen, die nach dem Medizinproduktegesetz geprüft wurden und als Medizinprodukte der Klasse I oder IIa eingestuft werden. Das Bundesinstitut für Arzneimittel und Medizinprodukte (BfArM) stellt auf seiner Internetseite (https://diga.bfarm.de/ de/verzeichnis) eine Liste der aktuell zu- 

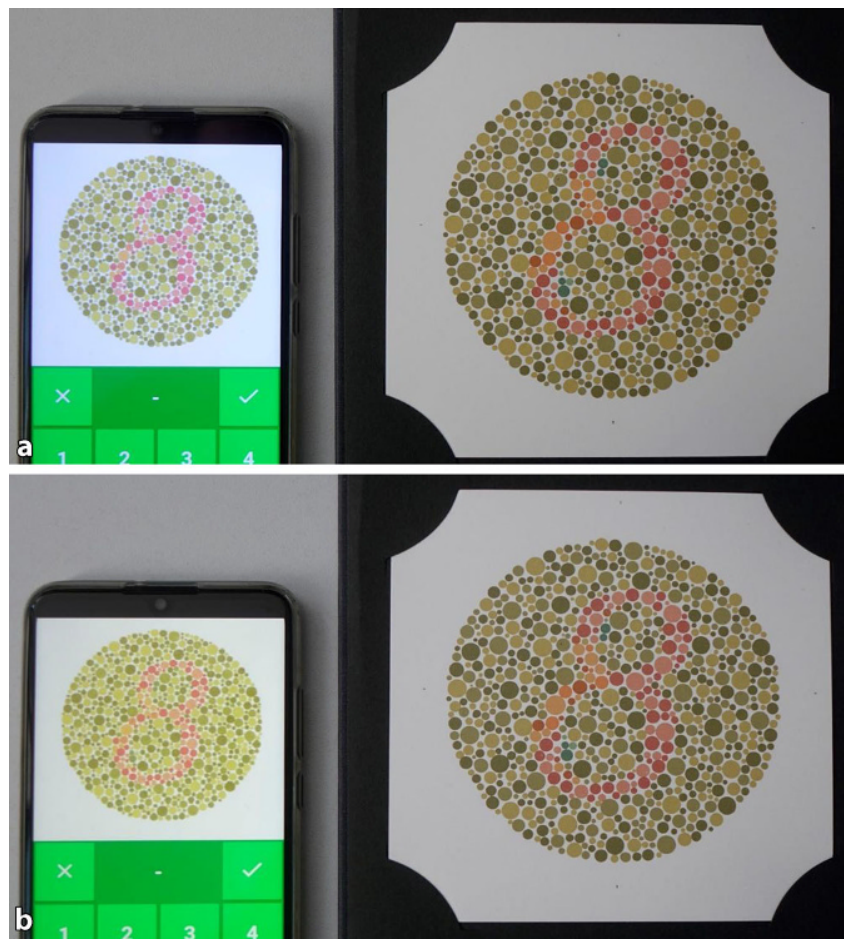

Abb. $3<$ Die Farbdarstellung digitaler Farbsehtests hängt von den Bildschirmeinstellungen $a b$. Im unteren Bild wurde der Blauanteil des SmartphoneBildschirms reduziert. Zum Vergleich ist jeweils rechts die Druckversion derselben Farbtafel eines international anerkannten Farbsehtests dargestellt. Die Farben weichen deutlich voneinander ab. a Handydisplay bei $80 \%$ Helligkeit und vollem Farbspektrum. b Handydisplay bei $80 \%$ Helligkeit und reduziertem Blauanteil im „Augenschonmodus" gelassenen DiGA zur Verfügung. Es finden sich darunter derzeit keine ophthalmologischen Anwendungen.

\section{Monitoring bei chronischen Augenerkrankungen}

Die einfachste Form des „remote/self monitoring" bei chronischen ophthalmologischen Erkrankungen ist sicher das Amsler-Gitter, das vornehmlich für die frühe Detektion der Konversion von trockener zu feuchter AMD, aber auch zur Erkennung anderer Netzhautpathologien der Makula verwendet wird. AmslerGitter gibt es auch für Smartphones (verschiedene Anbieter, kostenfrei in App-Stores). Diese haben den Vorteil, dass Skotome oder verzerrte Linien über den Touchscreen auf dem Amsler-Gitter eingezeichnet und die Bilder entweder lokal gespeichert oder an den Ophthalmologen digital übermittelt werden können. Gerade für die Beurteilung von Veränderungen im Verlauf kann dies von Interesse sein.

Neben den Netzhauterkrankungen ist v. a. das Glaukom als chronische Erkrankung Grund für regelmäßige Besuche beim Ophthalmologen. Sowohl der Augeninnendruck (IOD) als auch das Gesichtsfeld sollten regelmä- ßig überprüft werden. Telemedizinische Ansätze können hier evtl. zukünftig die Frequenz der Arztbesuche verringern und dabei gleichzeitig die Häufigkeit der Messungen erhöhen. So erlaubt z. B. ein für die Selbstmessung konstruiertes Rebound-Tonometer (iCare Home $^{\circledR}$, Icare Finland Oy, Vantaa, Finland), die eigenständige Messung des IOD durch den Patienten selbst. In einem multizentrischen Pilotprojekt unter der Leitung der Universitätsaugenklinik Münster (SALUS-Projekt, https://www. ukm.de/index.php?id=11854) sollen fast 2000 Patienten randomisiert entweder ein Tagesdruckprofil während eines Klinikaufenthaltes erhalten oder über 7 Tage mittels Selbsttonometrie Augendruckwerte erheben. Die solchermaßen gewonnenen Augendruckwerte sollen in einer elektronischen Fallakte gesammelt und den Patienten sowie deren behandelnden Augenärzten zur Einsicht zur Verfügung gestellt werden.

Eine Alternative dazu bietet ein implantierbarer intraokularer Drucksensor (eyemate ${ }^{\circledR}$, Implandata Ophthalmic Products $\mathrm{GmbH}$, Hannover), der berührungslose Selbstmessungen erlaubt und dessen Daten sich drahtlos an eine sichere Datenbank übertragen lassen [4]. Die Datenbank ist mit einer CE-zertifizierten cloudbasierten elektronischen Patientenakte gekoppelt, die unter anderem ein Modul zur Auswertung und Darstellung der telemetrischen Druckmessungen, aber auch manuell erhobener Druckwerte enthält (Medstage TonoTracker ${ }^{\circledR}$, Talkingeyes \& More GmbH, Erlangen). Das Modul kann IOD-Verläufe über verschiedene Zeiträume darstellen und aus den Selbstmessungen auch ein Tagesdruckprofil über einen gewählten Zeitraum rekonstruieren. Darüber hinaus lassen sich Verlauf und Tagesdruckprofil auch für verschiedene Zeiträume vergleichen, z.B. vor und nach therapeutischer Intervention (• Abb. 4). Während der COVID-19-Pandemie hat sich das System für Patienten, die mit dem Sensor ausgestattet sind, bereits als hilfreich erwiesen und zum Teil $\mathrm{zu}$ Therapieentscheidungen anhand der Selbstmessungen geführt [16].

\section{》) Bei Glaukom sollten Augeninnendruck und Gesichtsfeld regelmäßig überprüft werden}

Auch Gesichtsfeldkontrollen könnten zukünftig von Patienten zu Hause selbst durchgeführt werden. Jones et al. verglichen im Rahmen einer Studie an 20 Glaukompatienten konventionelle Gesichtsfelduntersuchungen am Anfang und Ende der Studie mit von den Patienten für ein halbes Jahr monatlich selbst durchgeführten Untersuchungen an einem Tablet-PC (Eyecatcher) in der häuslichen Umgebung. Sie fanden dabei sowohl eine hohe Adhärenz der Patienten als auch eine sehr hohe Konkordanz zwischen den Messverfahren $(\mathrm{r}=0,94, p<0,001)$. Die Qualität der Messbedingungen (korrekte Fixation, Umfeldbeleuchtung, Abstand zum Tablet) konnte darüber hinaus mittels Videoaufzeichnungen der Tablet-Kamera überprüft werden [12]. Eine auf Augenbewegungen basierende Variante des Eyecatcher stellten ebenfalls Jones et al. vor. Bei dieser werden mithilfe eines am Tablet-PC angebrachten Eyetrackers die Blickbewegungen hin $\mathrm{zu}$ den angebotenen Lichtstimuli erfasst, 
und daraus wird eine Art Gesichtsfeld rekonstruiert. Im Wartezimmer einer Glaukomsprechstunde konnten so bereits vor der Standardperimetrie Befunde von 77 Patienten erhoben werden, die gut mit denen der konventionellen Perimetrie übereinstimmten und dabei sowohl schneller erhoben werden konnten, als auch von den Patienten als angenehmer wahrgenommen wurden [13].

Neben Tablet-basierter Perimetrie gibt es auch Ansätze, die Lichtempfindlichkeit der Netzhaut mittels VirtualReality Brillen zu erfassen, mit teils sehr guter Übereinstimmung mit konventioneller Perimetrie [5, 19]. Da Virtual-Reality-Brillen derzeit nicht so weit verbreitet sind wie Tablet-PCs, werden sich diese vermutlich nicht so schnell für telemedizinische Anwendungen durchsetzen.

\section{Therapeutische und rehabilitative Ansätze}

Dieser Bereich bietet derzeit noch die wenigsten telemedizinischen Anwendungen. Vor allem dichoptische Spiele
(• Abb. 5) und Videos mit dem Ziel der Behandlung einer Amblyopie sind derzeit verfügbar, auch wenn der Nutzen solcher Anwendungen noch Gegenstand der Forschung ist $[2,7]$.

Denkbar wäre auch ein computerunterstütztes Training der exzentrischen Fixation bei Makuladegeneration $[8,11]$ oder das Üben von visuellen Suchstrategien und systematischen Blickrichtungswechseln, insbesondere bei Gesichtsfelddefekten. Verschiedene Kliniken und Praxen nutzen z. B. bereits eine Software zum Sakkadentraining bei Hemianopsie oder Retinopathia pigmentosa (www. visiocoach.de). Computerbasierte vergrößernde Sehhilfen werden im LowVision-Bereich bereits erfolgreich eingesetzt. Trainingsverfahren mithilfe von Smart-Devices können hier potenziell das therapeutisch/rehabilitative Spektrum erweitern.

\section{Ophthalmologische Video- sprechstunde}

Videosprechstunden sind bereits seit mehreren Jahren durch das E-HealthGesetz erlaubt. Sie werden derzeit insbesondere von radiologischen und Allgemeinarztpraxen vornehmlich zur Befundbesprechung eingesetzt. Auch psychologische Sprechstunden und Therapiesitzungen werden in steigender Zahl online angeboten.

Spätestens seit 2018 sind Videosprechstunden jedoch nicht nur für Befundbesprechungen, sondern auch für den Erstkontakt mit vorher nicht in der Praxis/Klinik bekannten Patienten erlaubt. Grundlage für Videosprechstunden ist die Nutzung eines zertifizierten Videodienstanbieters, dessen Verwendung auch der zuständigen Kassenärztlichen Vereinigung angezeigt werden muss, um als ärztliche Leistung abgerechnet werden zu können. Bislang wurden Videodienste jedoch nur von wenigen Praxen und Kliniken genutzt, unter anderem weil das elektronische

Hier steht eine Anzeige. 


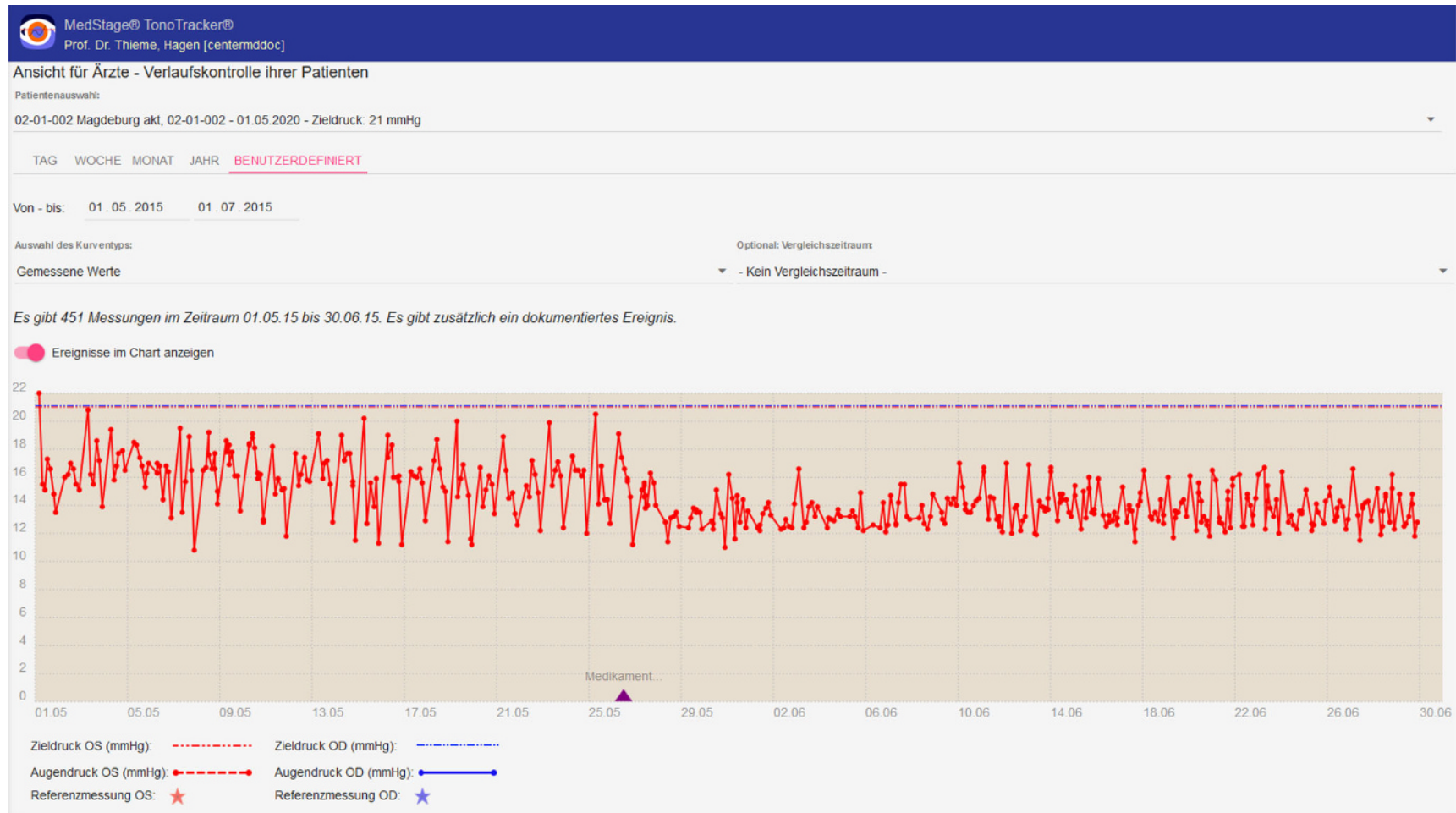

Abb. 4 \ Screenshot der TonoTracker-Anwendung. Dargestellt sind die vom Patienten mittels Eyemate selbst gemessenen IOD-Werte über einen Zeitraum von 2 Monaten inklusive Markierung des Zeitpunkts eines Medikamentenwechsels. (๑ Medstage TonoTracker ${ }^{\circledR}$, Talkingeyes \& More $\mathrm{GmbH}$, Erlangen)

Rezept und die elektronische Arbeitsunfähigkeitsbescheinigung derzeit noch nicht implementiert sind. Pilotprojekte laufen erst Mitte 2021 an, und beides wird erst ab Januar 2022 verpflichtend, und so ist eine vollständige technische Umsetzung einer reinen Online-Sprechstunde aktuell noch schwierig. Zudem sind die verfügbaren Videodienste bislang in den wenigsten Fällen in eine der vielen Praxis-Software-Plattformen integriert. Die Kassenärztliche Bundesvereinigung listet derzeit 44 zertifizierte Videodienste (Stand Oktober 2020) auf ihrer Homepage (https://www.kbv.de/ $\mathrm{html} /$ videosprechstunde.php), und diese große Auswahl macht die Entscheidung zur Durchführung einer Videosprechstunde sicher nicht einfacher. Dabei ließe sich gerade in der Augenheilkunde mittels Videosprechstunde eine Triage der Patienten organisieren, um dringend behandlungsbedürftige Patienten frühzeitig zu identifizieren und andererseits in Bagatellfällen dem Patienten den Weg zum Augenarzt zu ersparen. Die Akzep$\tan z$ solcher Videokonsultationen bei
Patienten war in Pilotprojekten während der COVID-19-Pandemie hoch [9].

\section{》) Grundlage für Videosprech- stunden ist die Nutzung eines zertifizierten Videodienstanbie- ters}

Zwar kann eine ophthalmologische Videosprechstunde die Spaltlampenuntersuchung nicht ersetzen, sie bietet vielleicht besser als in anderen Fachgebieten die Möglichkeit, zumindest orientierende Funktionsüberprüfungen des Sehsystems durchführen. So ließen sich die oben beschriebenen Screeningund Monitoringanwendungen in $\mathrm{Zu}$ kunft mit wenig technischem Aufwand in die Sprechstunde einbinden, angefangen bei Visustests (inklusive „preferential looking“) über Farbsehtests hin zur Prüfung des zentralen Gesichtsfelds. Auch einfache strabologische Tests (z.B. Motilitätstests, Hirschberg-Test über das Leuchten des Monitors in Kombination mit der Webcam, Abdecktest durch den
Patienten selbst, Untersuchungen zum Binokularsehen mithilfe von Anaglyphenbrillen) ließen sich am Bildschirm durchführen.

\section{Diskussion}

Der hier gezeigte Überblick über aktuell verfügbare und z.T. noch in der Entwicklung befindliche Ansätze zur telemedizinischen Anwendung belegt, dass die Ophthalmologie von digitalen $\mathrm{Ge}$ sundheitsanwendungen in Zukunft stark profitieren könnte. Die Warteräume von Praxen und Kliniken könnten entlastet werden, und insbesondere Patienten mit eingeschränkter Mobilität könnte ein Teil der Augenarztbesuche vielleicht erspart bleiben. Auch ophthalmologisch unterversorgte Einrichtungen (z. B. Seniorenheime) $[6,14]$ und ländliche Regionen, in denen ophthalmologische Praxen z. T. nicht besetzt sind, könnten mithilfe digitaler Anwendungen besser an die augenärztliche Versorgung angebunden werden. 


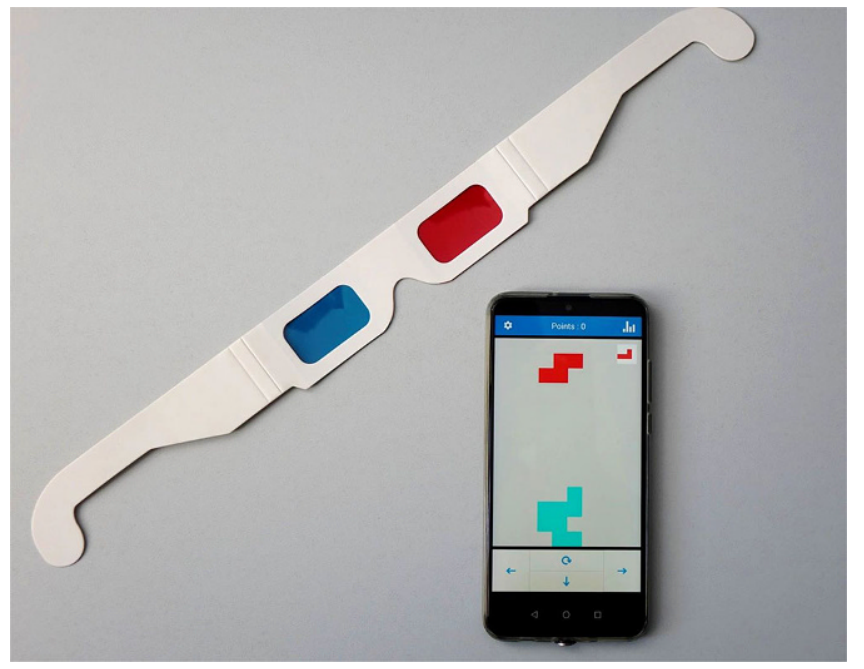

Abb. 5 A Dichoptisches, Tetris-ähnliches Spiel mit Anaglyphenbrille zur Amblyopiebehandlung. Die dargestellte Anwendung ist nicht CE-zertifiziert. Warnhinweise bezüglich Auftreten von Doppelbildern bei Strabismuspatienten finden sich nur auf der Homepage des Herstellers (von der App aus aufrufbar). Dort findet sich auch der Hinweis, dass es sich bei der App nicht um ein Medizinprodukt handelt

\section{》) Die Ophthalmologie könnte von digitalen Gesundheitsanwendungen in Zukunft stark profitieren}

Es zeigt sich aber auch, dass es derzeit ein noch ungenügendes Angebot zertifizierter und validierter digitaler Gesundheitsanwendungen für solche Einsatzzwecke gibt, die bereits jetzt für klinisch sichere Verwendung geeignet wären. Es besteht deshalb aktuell ein großer Forschungsund Entwicklungsbedarf auf diesem Gebiet. Zugleich bietet die pandemiebedingte Sensibilisierung für das Thema Telemedizin die Chance, die Entwicklung entscheidend voranzubringen. Die ophthalmologischen Fachverbände (z. B. DOG [Deutsche Ophthalmologische Gesellschaft] und BVA [Berufsverband der Augenärzte Deutschlands e. V.]) können hier sowohl auf politischer Ebene Einfluss nehmen als auch eine qualifizierte Bewertung aktuell verfügbarer Anwendungen bereitstellen, um den Augenärzten die Auswahl digitaler Hilfsmittel zu erleichtern. Darüber hinaus könnten sie auch durch gezielte Unterstützung bei der Entwicklung, Validierung und Zertifizierung von ophthalmologischen digitalen Anwendungen für den telemedizinischen Einsatz mithelfen. Ziel könnte beispielsweise die Bereitstellung einer spezialisierten Videosprechstundenplattform sein, in die validierte und zertifizierte ophthalmologische Funktionstests bereits fest integriert sind und die den Ansprüchen an das Medizinproduktegesetz und die europäische Datenschutzgrundverordnung genügt. Ein erster Schritt auf diesem Weg wäre die systematische Erfassung, in welchem Umfang telemedizinische Hilfsmittel von Augenärzten in Deutschland bereits genutzt werden, beispielsweise durch Abfrage der Zahl der bei den Kassenärztlichen Vereinigungen gemeldeten Videosprechstunden oder wissenschaftliche Auswertung entsprechender Umfragen unter den Augenärzten.

Ein wichtiger Nebeneffekt der zunehmenden Verbreitung von Screening- und Monitoring-Apps der Augenheilkunde könnte dabei auch die Verbesserung der epidemiologischen Forschung sein, da sich digitale Daten für solche Fragestellungen gut zusammenführen und auswerten lassen.

\section{Fazit für die Praxis}

- Die stetig wachsende Rechenleistung sowie die Qualität der Sensoren und Kameras von Computern und "smart devices" erlauben die Implementierung von telemedizinischen Anwendungen, die sowohl zum Screening als auch zum Monitoring bei chronischen Erkrankungen, evtl. sogar zu therapeutischen Zwecken eingesetzt werden können.

- Insbesondere älteren, immobilen Patienten kann so möglicherweise ein Gang zum Arzt erspart bleiben.

- Die Fähigkeit zur Übertragung digitaler Daten erlaubt zusätzlich Fortschritte hinsichtlich epidemiologischer und Versorgungsforschung.

- Gleichzeitig bergen solche Anwendungen aber auch Risiken, die z. B. die Gewährleistung des Schutzes von persönlichen und gesundheitsbezogenen Daten betreffen und einen verantwortungsbewussten Umgang mit bereits verfügbaren digitalen Hilfsmitteln erfordern.

- Eine zertifizierte Plattform für ophthalmologische Videosprechstunden, die validierte Screening- und Monitoring-Apps integriert, könnte zukünftig die Versorgung ophthalmologischer Patienten verbessern und Praxen und Kliniken entlasten.

\section{Korrespondenzadresse}

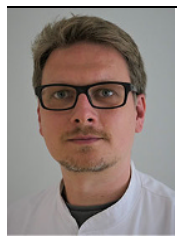

Dr. med. Lars Choritz

Medizinische Fakultät, Ottovon-Guericke Universität, Universitätsaugenklinik Leipziger Str. 44, 39120 Magdeburg, Deutschland lars.choritz@med.ovgu.de

\section{Einhaltung ethischer Richtlinien}

Interessenkonflikt. L. Choritz, M. Hoffmann und H. Thieme geben an, dass kein Interessenkonflikt besteht.

Für diesen Beitrag wurden von den Autoren keine Studien an Menschen oder Tieren durchgeführt. Für die aufgeführten Studien gelten die jeweils dort angegebenen ethischen Richtlinien.

\section{Literatur}

1. Arnold RW, O'Neil JW, Cooper KL et al (2018) Evaluation of a smartphone photoscreening app to detect refractive amblyopia risk factors in children aged 1-6 years. Clin Ophthalmol 12:1533-1537. https://doi.org/10.2147/OPTH.S171935

2. Bach M (2016) Dichoptic training for amblyopia. Ophthalmologe 113:304-307. https://doi.org/10. 1007/s00347-016-0238-4 
3. Brucker J, Bhatia V, Sahel J-A et al (2019) Odysight: a mobile medical application designed for remote monitoring-a prospective study comparison with standard clinical eye tests. Ophthalmol Ther 8:461-476. https://doi.org/10.1007/s40123-0190203-9

4. Choritz L, Mansouri K, van den Bosch J et al (2019) Telemetric measurement of intraocular pressure via an implantable pressure sensor-twelvemonth results from the ARGOS-02 trial. Am J Ophthalmol. https://doi.org/10.1016/j.ajo.2019. 09.011

5. Deiner MS, Damato BE, Ou Y (2020) Implementing and monitoring at-home virtual reality oculo-kinetic perimetry during COVID-19. Ophthalmology 127:1258. https://doi.org/10.1016/j.ophtha.2020. 06.017

6. Fang PP, Schnetzer A, Kupitz DG et al (2017) Ophthalmologische Versorgung in Seniorenheimen. Ophthalmologe 114:818-827. https://doi.org/10. 1007/s00347-017-0557-0

7. Foss AJE (2017) Use of video games for the treatment of amblyopia. Curr Opin Ophthalmol 28:276-281. https://doi.org/10.1097/ICU. 0000000000000358

8. Gaffney AJ, Margrain TH, Bunce CV, Binns AM (2014) How effective is eccentric viewing training? A systematic literature review. Ophthalmic Physiol Opt 34:427-437. https://doi.org/10.1111/opo. 12132

9. Gerbutavicius R, Brandlhuber U, Glück $S$ et al (2020) Evaluation of patient satisfaction with an ophthalmology video consultation during the COVID-19 pandemic. Ophthalmologe. https://doi. org/10.1007/s00347-020-01286-0

10. Gurovich Y, Hanani Y, Bar O et al (2019) Identifying facial phenotypes of genetic disorders using deep learning. Nat Med 25:60-64. https://doi.org/10. 1038/s41591-018-0279-0

11. Hassan SE, Ross NC, Massof RW, Stelmack J (2019) Changes in the properties of the preferred retinal locus with eccentric viewing training. Optom Vis Sci 96:79-86. https://doi.org/10.1097/OPX. 0000000000001324

12. Jones PR, Campbell P, Callaghan T et al (2020) Glaucoma home-monitoring using a tablet-based visual field test (Eyecatcher): an assessment of accuracy and adherence over six months. Am J Ophthalmol. https://doi.org/10.1016/j.ajo.2020. 08.039

13. Jones PR, Lindfield D, Crabb DP (2020) Using an open-source tablet perimeter (Eyecatcher) as a rapid triage measure for glaucoma clinic waiting areas. Br J Ophthalmol. https://doi.org/10.1136/ bjophthalmol-2020-316018

14. Larsen PP, Thiele S, Krohne TU et al (2019) Visual impairment and blindness in institutionalized elderly in Germany. Graefes Arch Clin Exp Ophthalmol 257:363-370. https://doi.org/10. 1007/s00417-018-4196-1

15. Livingstone I, Bastawrous A, Giardini ME et al (2014) Peek: portable eye examination kit. The Smartphone ophthalmoscope. Invest Ophthalmol Vis Sci 55:1612-1612

16. Mansouri K, Kersten-Gomez I, Hoffmann EM et al (2020) Intraocular pressure telemetry for managing glaucoma during the COVID-19 pandemic. Ophthalmol Glaucoma. https://doi. org/10.1016/j.ogla.2020.12.008

17. Mishima H, Suzuki H, Doi M et al (2019) Evaluation of Face2Gene using facial images of patients with congenital dysmorphic syndromes recruited in Japan. J Hum Genet 64:789-794. https://doi.org/ 10.1038/s10038-019-0619-z
18. Munson MC, Plewman DL, Baumer KM et al (2019) Autonomous early detection of eye disease in childhood photographs. Sci Adv 5:eaax6363. https://doi.org/10.1126/sciadv.aax6363

19. Razeghinejad R, Gonzalez-Garcia A, Myers JS, Katz LJ (2021) Preliminary report on a novel virtual reality perimeter compared with standard automated perimetry. J Glaucoma 30:17-23. https://doi.org/10.1097/IJG.0000000000001670

20. Steren BJ, Young B, Chow J (2021) Visual acuity testing for telehealth using mobile applications. JAMA Ophthalmol. https://doi.org/10.1001/ jamaophthalmol.2020.6177
DOG

Deutsche Ophthalmologische Gesellschaft

Gesellschaft

für Augenheilkunde

\section{Bereich für Mitglieder der DOG auf SpringerMedi- zin.de}

Schauen Sie sich den neuen Bereich für Mitglieder der DOG auf SpringerMedizin.de an: www.springermedizin.de/dog

Hier finden Sie

- die Leitlinien, Empfehlungen und Stellungnahmen der DOG auf einen Blick,

- schnellen Zugriff auf die CME-Kurse von Der Ophthalmologe,

- das Online Archiv von Der Ophthalmologe rückwirkend bis 1997,

- die aktuelle Ausgabe als e.Paper zum Offline-Lesen unterwegs,

- direkte Links zur Webseite der DOG und zum DOG E-Campus.

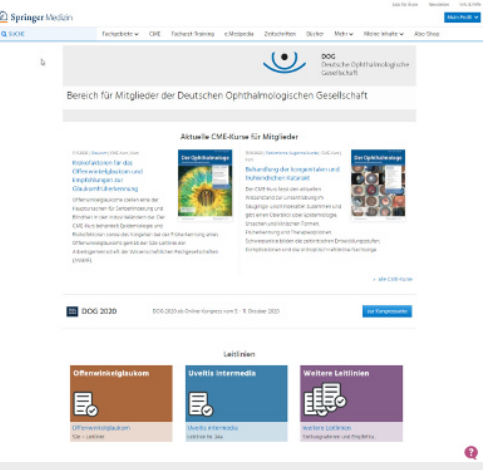

Registrieren Sie sich einmal kostenfrei auf www.springermedizin.de. Geben Sie dabei Ihren Vor- und Nachname und Lieferadresse wie auf dem Adressaufkleber auf Ihrem Heft an. So kann im System die Zugehörigkeit zur DOG sichergestellt werden.

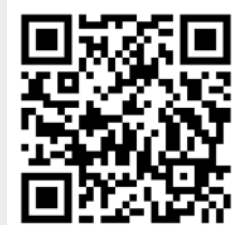

Über diesen QR-Code schnell

und einfach lossurfen 